\title{
Breast Reconstruction After Radiotherapy
}

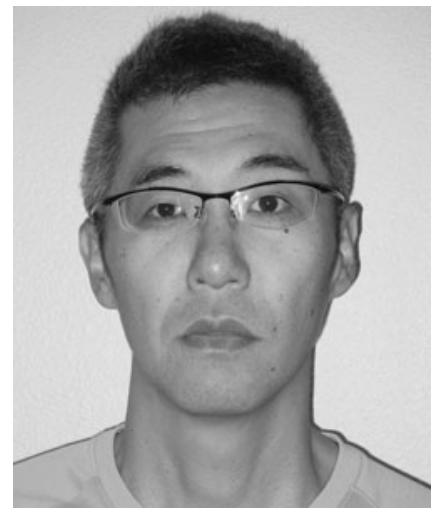

Hiroshi Yoshimoto, MD

Submitted for publication December 1, 2012 Accepted in revised form January 20, 2013

${ }^{*}$ Correspondence: Department of Plastic and Reconstructive Surgery, Nagasaki University Hospital, 1-7-1 Sakamoto, Nagasaki City 852-8501, Japan (e-mail: hy671117@nagasaki-u.ac.jp).

\footnotetext{
Abbreviations and Acronyms

ADRCs $=$ adipose-derived regenerative cells

ADSCs $=$ adipose-derived stem cells

DIEP = deep inferior epigastric perforator

$\mathrm{LD}=$ lattissimus dorsi musculocutaneous

TRAM $=$ transverse rectus abdominis myocutaneous
}

\author{
Hiroshi Yoshimoto* and Rodrigo Hamuy \\ Department of Plastic and Reconstructive Surgery, Nagasaki University Hospital, Nagasaki City, Japan.
}

It was once common practice in the treatment of breast cancer for total mastectomy and axillary lymph node dissection to be conventionally performed. However, breast-conserving surgery is increasingly being performed with marked improvement in a multidisciplinary treatment approach, including surgery, chemotherapy, irradiation, and antihormonal therapy. We must choose the optimal reconstructive methods with the reduction of the excision range. Furthermore, we also consider the local chronic radiation injury after adjuvant radiotherapy on breast reconstruction. As for breast reconstruction, the use of autologous tissues or artificial prostheses is common. However, after radiotherapy or if radiotherapy is planned, the complications such as infection, skin necrosis, or exposure of the implant are increased in breast reconstruction with implants. Therefore, the breast is reconstructed with autologous tissue mainly with radiotherapy using an autologous flap transfer. Meanwhile, the autologous fat transfer with adipose-derived regenerative cells for repair and regeneration has recently been investigated in reconstructive surgery. We discuss the autologous flap and fat transfer for breast reconstruction.

\section{BACKGROUND Flap transfer}

AN AUTOLOGOUS FLAP IS EFFECTIVE for irradiated damaged tissue in breast reconstruction because the blood supply of the flap is abundant. The transverse rectus abdominis myocutaneous (TRAM) flap is one of the most popular methods for breast reconstruction. Recently, with the development of microsurgery, a lot of perforator flaps with minimal donor-site morbidity had been developed. ${ }^{1}$ The deep inferior epigastric perforator (DIEP) flap has been used more often for breast reconstruction from the abdomen with the preservation of the rectus muscle as opposed to the TRAM flap. Postoperative pain is reduced, and abdominal hernia is very rare because of minimal muscle or fascial scarification. The DIEP flap has other advantages. The tissue vol- ume of the DIEP flap is sufficient to reconstruct the breast mound. The fat in the lower abdomen is soft and easy to shape. Therefore, the DIEP flap is suitable for the breast reconstruction. On the other hand, the DIEP flap has also some disadvantages. Microvascular anastomosis is required with adequate training. If the anastomosis fails, the flap will necrose unless the cause is solved.

The surgical procedure is more difficult than the TRAM flap, and the blood and nerve supply of the rectus muscle must be preserved, and the anastomosed vessels of the DIEP flap are thinner.

Furthermore, the superficial inferior epigastric artery or gluteal artery perforator flaps are also used as other perforator flaps for breast reconstruction with more tissue conservation. ${ }^{1}$ 


\section{Fat transfer}

Even though autologous fat transfer is an older method for correcting soft tissue defects, much of the injected fat will be reabsorbed, causing scar tissue and calcifications. In 1987, the Society of Plastic and Reconstructive Surgeons issued a position statement by which the society "strongly condemns the use of fat injections for breast enlargement," warning that the procedure "may hamper the detection of early breast cancer or result in false-positive cancer screening."

Thereafter, the results of autologous fat transfer have improved with advanced techniques of liposuction and injection. Furthermore, there are several reports that the survival rate has increased with the addition of platelet-rich plasma, ${ }^{2}$ insulin, insulin-like growth factor I, or basic fibroblast growth factor ${ }^{3}$ to the fat. Recently, there is a growing interest in adipose-derived regenerative cells (ADRCs). ${ }^{4}$ ADRCs can be harvested with a minimally invasive procedure by liposuction through small incisions (Fig. 1a). ADRCs contain several types of stem and regenerative cells, including adipose-derived stem cells (ADSCs), endothelial and smooth muscle cells and their progenitors, and preadipocytes. ADSCs have the capacity to differentiate into multiple lineages such as fat, bone, cartilage, endothelial cells, and other types of cells. ADSCs secrete various types of cytokines and growth factors that promote neovascularization and modulate the immune reaction. Since there is a greater amount of harvesting tissue and higher count of stem cell populations in than those of bone marrow-derived mesenchymal stem cells, ADRCs can be used as a noncultured cell source. Thus, ADRC stem cell therapy can be performed simultaneously at the time of surgical debridement in the operation ward (Figs. 1 and 2). The first prospective clinical trial (RESTORE-2) was performed with the use of ADRC-enriched fat grafts to treat breast defects post-breast conservation therapy with or without radiation. ${ }^{5}$ About 67 patients were treated. Patients $(75 \%)$ and investigators $(85 \%)$ were satisfied with treatment results after 12 months. The breast contour of patients (83\%) improved by a blinded assessment of MRI images. There were no serious adverse events, and was no local cancer recurrence.

\section{DISCUSSION}

Tissue expander and implant-based have become the most popular form of breast reconstruction. Recently, the use of human acellular dermis has been incorporated into tissue expander and implant-based breast reconstruction as the
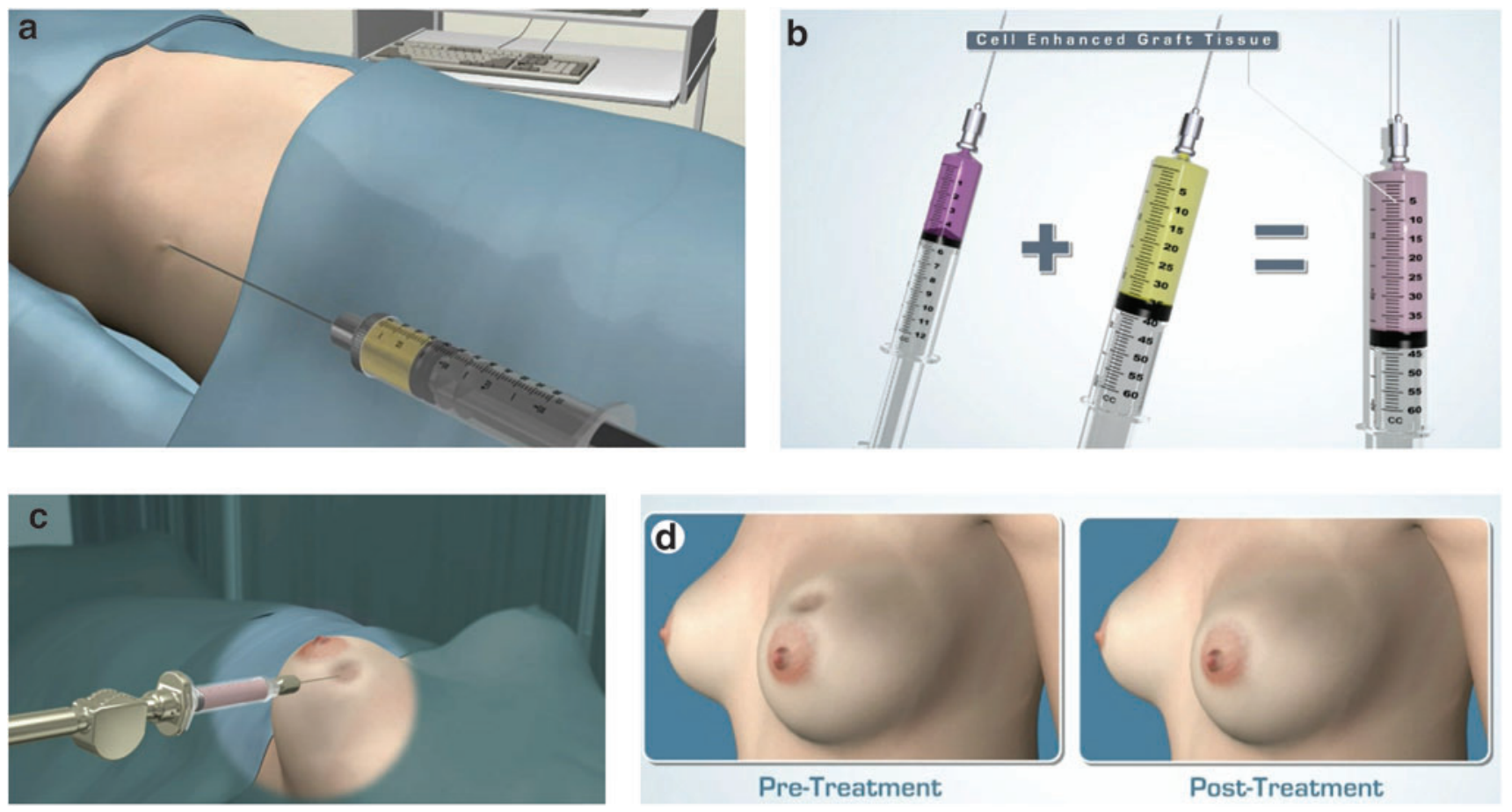

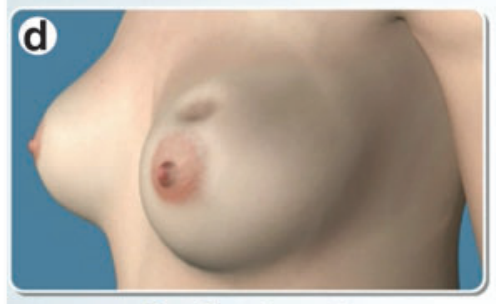

Pre-Treatment

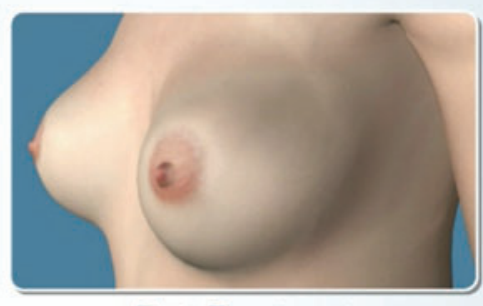

Post-Treatment

Figure 1. (a) Liposuction procedure. (b) Adipose-derived regenerative cells (ADRCs) and fat tissue are mixed for transplantation. (c) The mixture of ADRCs and fat tissue is injected to the breast defect. (d) Pretreatment view shows breast deformity. Post-treatment view: The defect has improved after fat transfer with ADRCs. Images provided by Cytori Therapeutics, Inc. (San Diego, CA). To see this illustration in color, the reader is referred to the web version of this article at www.liebertpub.com/wound 

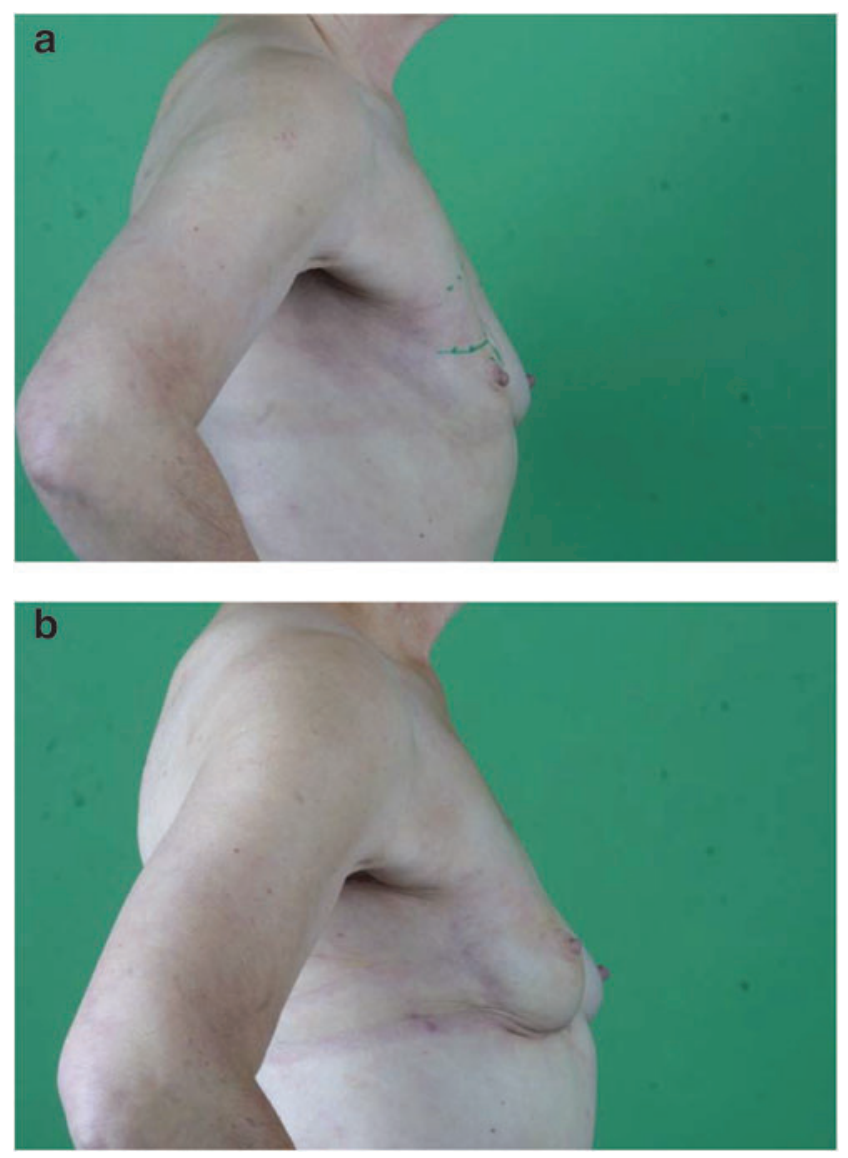

Figure 2. (a) A 58-year-old patient after mastectomy and radiotherapy had a persistent deformity after reconstruction with a transverse rectus abdominis myocutaneous flap and a lattissimus dorsi musculocutaneous flap. (b) The defect has improved after fat transfer with ADRCs. Reproduced with permission from Herold et al. ${ }^{4}$ To see this illustration in color, the reader is referred to the web version of this article at www.liebertpub.com/wound

pectoralis extender. However, this reconstruction method demonstrated high rates of clinically significant capsular contracture with radiotherapy. ${ }^{6}$

For breast reconstruction after radiotherapy or if radiotherapy is planned, autologous wellvascularized tissue, such as musculocutaneous, free, or local flaps, are suitable. However, the problem of tissue transplantation is the donor-site morbidity. The perforator flaps have less donor-site morbidity; however, linear scar certainly remains at the donor site. Since fat tissue and ADRCs can be harvested with a minimally invasive procedure by liposuction through small incisions, the scar is insignificant. There is an interesting report that compares the DIEP and lattissimus dorsi musculocutaneous (LD) flap regarding satisfaction with an aesthetic outcome between patients and plastic surgeons. ${ }^{7}$ The patients were more satisfied with the LD flap than DIEP flap reconstruction, which may relate to the donor-site scar. Surgeons favored DIEP flap reconstruction in regard to the resulting size and shape of the breast.

The surgical procedure of flap reconstruction is technically much more difficult than the fat transfer procedure. Adequate training and proper equipment are necessary for good results in flap reconstruction.

The grafted tissue volume could be adjusted according to the volume of defects in flap reconstruction. DIEP flap can additionally be adapted to large defects. However, the fatty tissue in free flap is reduced in thickness by about $16 \%$ in the nonirradiated group and about $21 \%$ in the irradiated group over a period of $6-10$ months after surgery. ${ }^{8}$ Surgeons should take notice of postoperative flap atrophy to obtain a good result. The survival rate for grafted fat tissue also could not be predicted in fat transfer with ADRCs. In RESTORE-2, patients with partial mastectomy defects of up to $150 \mathrm{~mL}$ were eligible for enrollment. Fat transfer with ADRCs is better than flap reconstruction to provide a better shape and fine contour. The fat transfer with ADRCs is also available for local radiation injury. ${ }^{9}$ Therefore, the fat transfer with ADRCs is effective for small or partial defects post-breast conservation therapy with radiotherapy. However, several questions about $\mathrm{ADRCs}$ still remain to be answered. Which cells do transplanted ADRCs differentiate into? What is the survival rate for transplanted ADRCs? Do transplanted ADRCs have an influence on the recurrence of tumor cells? Further studies are needed to assess fat transplantation with ADRCs for breast reconstruction. Fat transfer with ADRCs is considered to be one of the great options for breast reconstruction postbreast conservation therapy with radiotherapy.

\section{ACKNOWLEDGMENTS AND FUNDING SOURCES}

Images in Figure 1 were provided by Cytori Therapeutics, Inc. (San Diego, CA). Figure 2 is reproduced with permission from Herold et al. ${ }^{4}$

\section{AUTHOR DISCLOSURE AND GHOSTWRITING}

The authors have no commercial associations to disclose. This article is not written by any writer other than the authors listed.

\section{ABOUT THE AUTHORS}

Hiroshi Yoshimoto, $\mathbf{M D}, \mathbf{P h D}$, is an assistant professor in the Department of Plastic and Reconstructive Surgery at Nagasaki University Hospital. Hamuy O.L. Rodrigo, MD, graduated from a doctoral course of medical science at Nagasaki University in September 2013. 


\section{REFERENCES}

1. Tseng CY and Lipa JE: Perforator flaps in breast reconstruction. Clin Plast Surg 2010; 37: 641; vi.

2. Nakamura $S$, Ishihara $M$, Takikawa M, Murakami K, Kishimoto S, Nakamura S, Yanagibayashi S, Kubo S, Yamamoto N, and Kiyosawa T: Plateletrich plasma (PRP) promotes survival of fat-grafts in rats. Ann Plast Surg 2010; 65: 101.

3. Yuksel E, Weinfeld AB, Cleek R, Wamsley $S$, Jensen J, Boutros S, Waugh JM, Shenaq SM, and Spira M: Increased free fat-graft survival with the long-term, local delivery of insulin, insulin-like growth factor-I, and basic fibroblast growth factor by PLGA/PEG microspheres. Plast Reconstr Surg 2000; 105: 1712.

4. Herold C, Vogt PM, Busche MN, Jokuszies A, Park $\mathrm{J}$, and Rennekampff HO: Supplementation of fat grafts with adipose-derived regenerative cells in reconstructive surgery. GMS Germ Plast Reconstr Aesthetic Surg 2012; 2: Doc07: www.egms.de/ static/en/journals/gpras/2012-2/gpras000009.shtml

5. Pérez-Cano R, Vranckx JJ, Lasso JM, Calabrese C, Merck B, Milstein AM, Sassoon E, Delay E, and Weiler-Mithoff EM: Prospective trial of adiposederived regenerative cell (ADRC)-enriched fat grafting for partial mastectomy defects: the RESTORE-2 trial. Eur J Surg Oncol 2012; 38: 382.

6. Spear SL, Seruya M, Rao SS, Rottman S, Stolle E, Cohen M, Rose KM, Parikh PM, and Nahabedian MY: Two-stage prosthetic breast reconstruction using AlloDerm including outcomes of different timings of radiotherapy. Plast Reconstr Surg 2012; 130: 1

7. Lindegren $A$, Halle $M$, Docherty-Skogh $A C$, and Edsander-Nord A: Postmastectomy breast reconstruction in the irradiated breast: a comparative study of DIEP and latissimus dorsi flap outcome. Plast Reconstr Surg 2012; 130: 10.

8. Fujioka M, Masuda K, and Imamura Y: Fatty tissue atrophy of free flap used for head and neck reconstruction. Microsurgery 2011; 31: 32

9. Akita S, Yoshimoto H, Akino K, Ohtsuru A, Hayashida K, Hirano A, Suzuki K, and Yamashita $S$ : Early experiences with stem cells in treating chronic wounds. Clin Plast Surg 2012; 39: 281. 